\title{
URETER ECTÓPICO EN PEDIATRÍA; UN CAMBIO EN SU FORMA DE PRESENTACIÓN
}

José Manuel Escala, Yair Cadena González, Pedro J. López, Gabriela Retamal, Nelly Letelier y Ricardo Zubieta.

Unidad de Urología. Hospital Exequiel González Cortés. Santiago. Chile.

\begin{abstract}
Resumen.- OBJETIVO: Clásicamente el diagnóstico de uréter ectópico se hacía en niñas mayores por incontinencia urinaria, hoy los diagnósticos son más precoces, lo que ha cambiado parcialmente su tratamiento. El objetivo del presente trabajo es realizar una revisión de nuestra experiencia en los últimos años y correlacionar con la forma de presentación en la actualidad.
\end{abstract}

MÉTODOS: Se estudiaron todos los pacientes con diagnóstico de uréter ectópico en un periodo de 10 años, entre enero de 1997 y diciembre de 2006. Se analizaron las características demográficas, la forma de presentación, estudios realizados, edad al diagnóstico y tratamiento, junto a las distintas técnicas empleadas para solucionar su patología.
José Manuel Escala A.

Yair Cadena González

Unidad Urología Pediátrica

Hospital Exequiel González Cortes Barros Luco 3301 . San Miguel Santiago. (Chile). imescala@clinicalascondes.cl yaircadena@yahoo.com

Trabajo recibido: 3 de junio 2007.
RESULTADOS: Se encontraron 19 pacientes con esta patología, 15 de sexo femenino. La forma de presentación estuvo dada en 13 pacientes con infección urinaria febril, 2 con incontinencia urinaria y en 4 el diagnóstico fue prenatal por hidronefrosis.

Doble sistema pielo ureteral tenían 16 niños y solo 3 tenían sistema único. El diagnóstico en todos los casos se realizo con ecografía renal y vesical, uretrocistografía y estudios endoscópicos. Estudios adicionales como pielografía de eliminación se realizo en 8 al comienzo de la serie, y estudios de medicina nuclear en 17. La mediana de edad al momento del diagnóstico fue de 8 meses.

El tratamiento fue quirúrgico en todos. En los dobles sistemas se realizó heminefroureterectomía superior en 8 pacientes, reimplante vésicoureteral en 3 y píelo-pieloanastomosis en 3 casos con función remanente del polo renal superior. En otros 2 casos se realizó nefroureterectomía por presencia de reflujo al sistema inferior y compromiso renal severo. En los sistemas únicos se realizó reimplante en los 3 casos.

CONCLUSIONES: La sospecha prenatal y el estudio adecuado de las infecciones del tracto urinario permite confirmar el diagnóstico de uréter ectópico. Pocos niños debutan con incontinencia urinaria en la actualidad, debido a lo precoz del diagnóstico. El tratamiento es siempre quirúrgico, y depende básicamente de la función renal, y la presencia o no de relujo vésico ureteral. De preferencia en los pacientes con uréter ectópico en sistema único se realiza un reimplante vésicoureteral y en los con doble sistema una heminefrectomía superior, abandonando el uréter distal en una primera instancia.

Palabras clave: Uréter. Ectópico. Heminefrectomía. Incontinencia. 
Summary.- OBJECTIVES: Classically, the diagnosis of ectopic ureter was done in grown up girls due to urinary incontinence, today the diagnoses are more precocious, which has partially changed treatment. The objective of this paper is to perform a review of our experience over the last years and to correlate it with the current type of presentation.

METHODS: We studied all patients with the diagnosis of ectopic ureter in a period of 10 years, between 1997 and December 2006. Demographic characteristics, type of presentation, diagnostic tests performed, age at the time of diagnosis and treatment were all analyzed, altogether with the different techniques employed for treatment.

RESULTS: We found 19 patients with this disease, 15 of them females. Type of presentation was febrile urinary tract infection in 13 patients, urinary incontinence in two, and prenatal diagnosis of hydronephrosis in 4. Sixteen children had double pyeloureteral systems and only three had single systems. In all cases the diagnosis was performed with renal-bladder ultrasound, urethrocystogram and endoscopic studies. Additional studies such as excretion pyelograms were performed in 8 patients at the start of the series and nuclear medicine tests in 17. Median age at the time of diagnosis was eight months. All patients underwent surgical treatment. In patients with double systems superior heminephroureterectomy wae performed in 8 patients, vesicoureteral reimplantation in three and pyelo-pyelic anastomosis in three cases with upper pole remnant function. In another two cases nephroureterectomy was performed due to the presence of reflux to the lower or system and severe renal compromise. In all three cases with single systems ureter reimplantation was performed.

CONCLUSIONS: Currently prenatal suspicion and adequate study of urinary tract infections enable confirmation of ectopic ureter. Few children debut with urinary incontinence currently, due to the precocity of diagnosis. Treatment is always surgical, and basically depends on renal function, and the presence or absence of vesicoureteral reflux. Vesicoureteral reimplantation is performed preferentially in patients with ectopic ureter and a single system, and superior heminephrectomy in those with double systems, leaving the distal ureter of first option.

Keywords: Ureter. Ectopic. Heminephrectomy. Incontinence.

\section{INTRODUCCIÓN}

Clásicamente se define como uréter ectópico aquel que llega a cualquier lugar que no es el trígono en la vejiga, pero en general se acepta con éste término a todo uréter que tiene su meato en el cuello vesical o distal a él.
En los hombres en general éste llega sobre el esfínter externo, a nivel de uretra prostática, utrículo, vesículas seminales o deferentes por lo que su presentación es principalmente en forma de orquiepididimitis a repetición, sin embargo en las mujeres la llegada del uréter es bajo el esfínter, distal al cuello vesical por lo que determina una forma de presentación como incontinencia urinaria (1).

En los niños ésta patología ocurre con mucho mayor frecuencia en presencia de duplicación pieloureteral, y embriológicamente se produce por la presencia de un segundo brote ureteral que nace del mesonefros en posición anómala y no es incorporado en el trígono. Es poco frecuente en sistemas únicos, y suele asociarse a riñones displásicos (2), que pueden simular un riñón único.

El signo patognomónico para el diagnóstico de ésta patología ha sido el goteo permanente de orina en niñas a pesar de tener un patrón miccional normal después del control esfinteriano. Éste hecho seguido de un buen examen físico lograba el diagnóstico preciso en la mayoría de los casos. Para certificarlo bastaba una ecografía que demostrara el doble sistema, generalmente acompañado de una hidroureteronefrosis superior y estudios endoscópicos que confirmaban la posición anómala del meato (2).

En la actualidad vemos con menor frecuencia éste tipo de presentación, por lo que quisimos verificar la realidad actual de ésta patología en los últimos años en nuestra institución.

\section{MATERIAL Y MÉTODOS}

Desde enero de 1997 y diciembre del 2006 se revisaron las fichas médicas de todos los pacientes portadores de uréter ectópico intervenidos quirúrgicamente en forma retrospectiva.

Se analizó el sexo, la edad y forma de presentación, lateralidad, edad quirúrgica y tipo de cirugía realizada. Todos los pacientes cuentan con ecografía renal y vesical, uretrocistografía y estudios endoscópicos en los que se confirme el diagnóstico. Se analizaron las diferentes variables así como la presentación clínica de los pacientes, el tipo de manejo realizado y los resultados finales.

\section{RESULTADOS}

Un total de 15 pacientes de sexo femenino y 4 de sexo masculino portadores de uréter ectópico fueron intervenidos quirúrgicamente durante el perio- 
do de tiempo estudiado. De los 19 pacientes en total, en 16 el uréter ectópico estaba asociado a un doble sistema pielocaliceal y en 3 a sistemas únicos. En 12 se presento al lado izquierdo y 7 en el lado derecho.

La mediana de edad al diagnóstico fue a los 8 meses, de los cuales en 11 se realizó el diagnóstico en el primer año de vida, en 6 casos antes de los 2 años, y sólo en 2 casos en niños mayores.

La forma de presentación fue principalmente por sospecha antenatal en 4 casos, por estudio de infección urinaria en 13 y en sólo 2 por incontinencia urinaria.

Así mismo la mediana de edad quirúrgica fue de 15 meses y 14 de los 19 se operaron antes de los 2 años de vida.

Para el diagnóstico se realizó ecografía y uretrocistografía a todos, pielografía a 8, especialmente a los inicios de la serie y estudios de medicina nuclear en 17, para determinar la función del polo renal afectado.

Encontramos la asociación con reflujo al sistema inferior en 8 casos, de los cuales en 4 se reimplantaron los dos sistemas, en 2 se realizo nefroureterectomía por compromiso renal global, en un caso heminefrectomía superior y en otro ureteropieloanastomosis.

En cuanto al tratamiento de los 16 casos ocurridos en doble sistema, a 8 de ellos se les practicó una heminefroureterectomía, resecando el uréter en forma parcial, solo por la incisión subcostal en 7 y en uno se resecó en forma completa con una segunda incisión. Los restantes, con función del polo superior, en 3 se realizó una píelo-ureteroanastomosis, y los otros 3 fueron sometidos a un reimplante vésicoureteral de ambos uréteres juntos en cañón de escopeta con técnica de Gregoire, sin necesidad de afinamiento. En 2 casos se realizó nefroureterectomía por presentar función renal global disminuida. En los 3 pacientes con sistemas únicos se realizó un reimplante vésicoureteral con resección del segmento distal, usando la misma técnica.

La evolución de éstos pacientes ha sido buena, sólo uno requirió de una segunda cirugía, para resecar el muñón de uréter distal abandonado en la heminefrectomía, esto fue necesario, para tratar sus infecciones urinarias recurrentes, después de lo cual ha permanecido asintomático. Uno de los dos pacientes con diagnóstico tardío por incontinencia urinaria, persiste con una disfunción miccional importante por lo que ha requerido manejo médico adicional.

\section{DISCUSIÓN}

El uréter ectópico en la población infantil sigue siendo de baja ocurrencia y hay que sospecharlo claramente. Si definimos uréter ectópico como aquel que desemboca distal al trígono, probablemente son muchos que lo hacen entre el trígono y el cuello vesical, los que nunca dan sintomatología y por lo tanto no llegan a diagnosticarse. Campbell en 1970 encontró ésta patología en 10 niños sometidos a autopsia de un total de 19.700, por lo que su frecuencia se sitúa en alrededor de 1 por cada 2000 niños.

Sigue siendo una patología que ocurre prioritariamente en el sexo femenino (sólo 15\% en hombres, según Schulman, (1972) y dependiente del polo renal superior en portadores de doble sistema pieloureteral.

Lo que si ha cambiado con el tiempo es su forma de presentación, la forma clásica como cuadro de incontinencia, que sobrepasaba el $50 \%$ de ellos según Schlecker (1986) y otros, hoy ya casi no se ve, ya que se diagnostica en general antes que ocurra el control de esfínteres lo que hace mas difícil ésta forma de presentación. Es mas, la mayoría en el último tiempo se sospecha por hidronefrosis en ecografías maternas y el resto se diagnostica durante el estudio de sus infecciones urinarias, que suelen ocurrir con frecuencia en éstos niños a edades tempranas, por la ectasia que ocurre en el sistema comprometido (2).

El método de diagnóstico es a través de exámenes de imágenes, siendo la ecotomografía la de primera línea, donde se visualiza claramente la hidroureteronefrosis del polo superior, especialmente a nivel de pelvis renal, donde también se puede evaluar el grosor del parénquima, y en la zona baja donde se ve el uréter muy dilatado bajo la vejiga (3).

La uretrocistografía la usamos para certificar la presencia de un reflujo vésicoureteral, que pudiese ser relevante en la decisión quirúrgica posterior. El uso de la medicina nuclear, se ha hecho fundamental para obtener el conocimiento de la función renal del polo afectado. La pielografía en la actualidad se usa cada vez menos, solo en aquellos casos en que existe duda razonable de la anatomía. De todas maneras en nuestros pacientes el diagnóstico definitivo se realizo por la observación del meato ureteral ectópico durante la cistoscopia.

Respecto al tratamiento quirúrgico, éste depende de los exámenes descritos previamente, si no existe función aceptable del polo superior se prefiere realizar una heminefrectomía extirpando el uréter 
hasta donde sea posible y abandonando el extremo distal, éste da pocos problemas y no justifica su extirpación en el mismo acto quirúrgico (5-6) complicando la cirugía en tiempo y arriesgando el uréter sano o la continencia en un futuro al introducirse en la zona del cuello vesical (7). La resección del muñón se reserva para los casos que éste produce problemas, principalmente de tipo infecciosos en forma recurrente lo cual ocurrió en sólo uno de nuestros pacientes.

En la actualidad se está utilizando con frecuencia creciente la laparoscopia para éste tipo de intervenciones, lo que además sería de mucha utilidad en la extirpación del uréter en su totalidad en un solo tiempo con mayor facilidad como lo expresan diversos autores, ésta puede ser trans o retroperitoneal (8) y por vía lateral o posterior (9). La embolización arterial también ha sido mencionada recientemente como posible intervención (10).

Si nos encontramos frente a un caso con buena función renal del polo superior, su cirugía dependerá de la presencia o no de reflujo, si no tiene, elegimos una pieloureteroanastomosis, o uretero anastomosis para no intervenir en la zona cercana al cuello vesical, de lo contrario si tiene reflujo vésicoureteral, preferimos realizar un reimplante vésicoureteral en doble cañón. La píelo ureteroanastomosis ha demostrado ser una muy buena cirugía para la preservación de función renal (11).

Siguiendo ésta conducta hemos tenido resultados quirúrgicos buenos con preservación de función renal, y sin complicaciones mayores, siempre es importante realizar un seguimiento prolongado y adecuado de éstos pacientes.

Los casos que se presentan con incontinencia urinaria únicamente son cada vez mas escasos $(12,13)$, esto debido al aumento de la ecografía antenatal y al estudio adecuado de infecciones urinarias, lo que hace que estos pacientes de diagnostiquen $y$ se traten en una edad cada vez mas temprana.

\section{CONCLUSIONES}

El aumento de la ecografía antenatal y el estudio adecuado de las infecciones del tracto urinario, permite cada vez mas llegar a un diagnóstico oportuno y tratamiento temprano de esta patología que en épocas pasadas se diagnosticaba de una forma mas tardía y en muchas ocasiones por incontinencia urinaria. El estudio completo y cuidadoso de esta patología permite realizar un abordaje adecuado minimizando posibles complicaciones y procedimientos adicionales.

\section{BIBLIOGRAFÍA y LECTURAS RECOMENDADAS ( ${ }^{*}$ lectura de interés $y^{* *}$ lectura fundamental)}

1. SNYDER, H.S.; GILLENWATER, J.Y.; GRAYHACK, J.F.: "Adult and Pediatric Urology”. Vol 2. Chapter 48. Yearbook Medical Publisher Inc, Chicago, 1987.

2. CHOWDHARY, S.K.; LANDER, A.; PARASHAR, K. y cols.: "Single system ectopic ureter: A 15 years review”. Ped. Surg. Int., 17: 638, 2001.

3. VIJAYARAGHAVAN, S.B.: "Perineal sonography in diagnosis of an ectopic ureteric opening into the urethra". J. Ultras. Med., 21: 1041, 2002.

*4. BERROCAL, T.; LOPEZ-PEREIRA, P.; ARJONILLA, A. y cols.: "Anomalies of the distal ureter, bladder , and uretra in children: Embriologic, radiologic, and pathologic features". Radiographics, 22: 1139, 2002.

5. DE CALUWE, D.; CHERTIN, B.; PURI, P.: "Fate of the retained ureteral stump after upper pole heminephrectomy". J. Urol., 168: 679, 2002.

*6. ANDROULAKAKIS, P.A.; STEPHANIDIS, A.; CHRISTOPHORIDIS, C.: "Outcome of the distal ureteric stump after (hemi) nephrectomy and subtotal ureterectomy for reflux or obstruction". BJU Int., 88: 586, 2001.

7. ADE-AJAYI, N.; WILCOX, D.T.; DUFFY, P.G. y cols.: "Upper pole heminephrectomy: Is complete ureterectomy necessary?". BJU Int., 88: 77, 2001.

8. BORZI, P.A.; YEUNG, C.K.: "Selective approach for transperitoneal and extraperitoneal endoscopic nephrectomy in children”. J. Urol., 171: 814, 2004.

9. BORZI, P.A.: "A comparison of the lateral and posterior retroperitoneoscopic approach for complete and partial nephroureterectomy in children”. BJU Int., 87: 517, 2001.

10. KUDOH, K.; KADOTA, M.; NAKAYAMA, Y. y cols.: "Transcatheter arterial embolization therapy for a hypoplastic pelvic kidney with ectopic ureter to control incontinence". Cardiovasc. Interv. Radiol., 26: 479, 2003.

*11. CHOI, H.; OH, S.J.: "The management of children with complete ureteric duplication: Selective use of uretero-ureterostomy as a primary and salvage procedure". BJU Int., 86: 508, 2000.

*12. BORER, J.G.; BAURER, S.B.; PETERS, C.A. y cols.: "A single-system ectopic ureter draining an ectopic dysplastic kidney: Delayed diagnosis in the young female with continuos urinary incontinence". BJU, 81: 474, 1998.

*13. GREGORY, R.; HANSON, B.; JOHN, M. y cols.: "Diagnosis of ectopic ureter as a cause of urinary incontinente”. J. Ped. Urol., 3: 53, 2007. 